\title{
Association of Communication Between Hospital-based Physicians and Primary Care Providers with Patient Outcomes
}

\author{
Chaim M. Bell, MD, PhD, FRCP(C) ${ }^{1,2,3}$, Jeffrey L. Schnipper, MD, MPH ${ }^{4}$, \\ Andrew D. Auerbach, MD, MPH ${ }^{5}$, Peter J. Kaboli, MD, MS $S^{6}$, Tosha B. Wetterneck, MD', \\ David V. Gonzales, $M D^{8}$, Vineet $M$. Arora, $M D, M A^{9}$, James X. Zhang, $M S, P h D^{9}$, \\ and David O. Meltzer, MD, $P h D^{9}$
}

\begin{abstract}
'Departments of Medicine and Health Policy Management and Evaluation, University of Toronto, Toronto, ON, Canada; ${ }^{2}$ St. Michael's Hospital, Toronto, ON, Canada; ${ }^{3}$ Keenan Research Centre in the Li Ka Shing Knowledge Institute of St. Michael's Hospital, Toronto, Canada; ${ }^{4}$ BWF Hospitalist Service and Division of General Medicine, Brigham and Women's Hospital and Harvard Medical School, Boston, MA, USA; ${ }^{5}$ University of California-San Francisco, San Francisco, CA, USA; ${ }^{6}$ lowa City VA Medical Center and the University of lowa Carver College of Medicine, lowa City, IA, USA; 'University of Wisconsin School of Medicine and Public Health, Madison, WI, USA; ${ }^{8}$ University of New Mexico, Albuquerque, NM, USA; ${ }^{9}$ University of Chicago, Chicago, IL, USA.
\end{abstract}

BACKGROUND: Patients admitted to general medicine inpatient services are increasingly cared for by hospitalbased physicians rather than their primary care providers (PCPs). This separation of hospital and ambulatory care may result in important care discontinuities after discharge. We sought to determine whether communication between hospital-based physicians and PCPs influences patient outcomes.

METHODS: We approached consecutive patients admitted to general medicine services at six US academic centers from July 2001 to June 2003. A random sample of the PCPs for consented patients was contacted 2 weeks after patient discharge and surveyed about communication with the hospital medical team. Responses were linked with the 30-day composite patient outcomes of mortality, hospital readmission, and emergency department (ED) visits obtained through follow-up telephone survey and National Death Index search. We used hierarchical multi-variable logistic regression to model whether communication with the patient's PCP was associated with the 30-day composite outcome.

RESULTS: A total of 1,772 PCPs for 2,336 patients were surveyed with 908 PCPs responses and complete patient follow-up available for 1,078 patients. The PCPs for 834 patients (77\%) were aware that their patient had been admitted to the hospital. Of these, direct communication between PCPs and inpatient physicians took place for 194 patients (23\%), and a discharge summary was available within 2 weeks of discharge for 347 patients (42\%). Within 30 days of discharge, 233

This paper was presented at the Society for General Internal Medicine Annual Meeting in April 2006.

Received March 18, 2008

Revised October 27, 2008

Accepted November 10, 2008

Published online December 20, 2008
(22\%) patients died, were readmitted to the hospital, or visited an ED. In adjusted analyses, no relationship was seen between the composite outcome and direct physician communication (adjusted odds ratio 0.87, 95\% confidence interval $0.56-1.34$ ), the presence of a discharge summary $(0.84,95 \%$ CI $0.57-1.22)$, or PCP awareness of the index hospitalization (1.08, 95\% CI 0.73-1.59).

CONCLUSION: Analysis of communication between PCPs and inpatient medical teams revealed much room for improvement. Although communication during handoffs of care is important, we were not able to find a relationship between several aspects of communication and associated adverse clinical outcomes in this multi-center patient sample.

KEY WORDS: hospitalist care; continuity of care; physician communication.

J Gen Intern Med 24(3):381-6

DOI: $10.1007 / \mathrm{s} 11606-008-0882-8$

( ) Society of General Internal Medicine 2008

\section{BACKGROUND}

Patients admitted to general medical wards are increasingly being cared for by hospital-based physicians, or hospitalists. ${ }^{1-3}$ These clinicians specialize in general medical care of hospitalized patients and seldom see outpatients as primary care providers (PCPs). Upon discharge of their patients, hospitalbased physicians usually transfer care to the patient's usual PCP. ${ }^{4-6}$

There are many benefits to the hospitalist model. ${ }^{3,4}$ However, a major concern of the hospitalist movement relates to the separation of hospital and ambulatory care. ${ }^{7}$ This may result in patient care discontinuities because of a lack of PCP involvement in their patient's hospital care. Poor care continuity may become most evident when a patient is discharged from hospital because PCPs often are unaware that their patient was hospitalized and frequently do not 
receive discharge summaries. ${ }^{8,9}$ In addition, poor information transfer can result in potentially preventable adverse events. ${ }^{10}$ Communication between the hospital physician and PCP can begin when the patient is admitted, occur throughout the hospitalization, and aid in management after hospital discharge. Therefore, in the absence of a discharge summary, a PCP can still be involved in important information transfer through other, and often preferred, communication methods. ${ }^{11}$

We report the results of a study linking a survey of hospital physician communication with PCPs to important 30-day patient outcomes. Our objective was to determine whether PCP knowledge of their patient's hospital admission, receipt of a discharge summary, and direct communication with the inpatient medical team are associated with 30-day composite patient outcomes of death, hospital readmission, or emergency department (ED) visit.

\section{METHODS}

\section{Setting}

The study was part of a large, multi-center trial evaluating the effects of hospitalists on costs and outcomes in hospitalized general medicine patients at six academic medical centers throughout the US: Brigham and Women's Hospital (BWH) in Boston, Massachusetts; University of Chicago Medical Center (UC) in Chicago, Illinois; University of California San Francisco Medical Center (UCSF) in San Francisco, California; University of Iowa Hospitals and Clinics (UI) in Iowa City, Iowa; University of New Mexico Medical Center (UNM) in Albuquerque, New Mexico; and University of Wisconsin Hospital and Clinics (UW) in Madison, Wisconsin. ${ }^{12}$ Approval for our study was obtained from the Institutional Review Boards at all six participating sites.

\section{Patients}

Our study population included consecutive patients providing informed consent and physician contact information from the 31,891 patients admitted from July 2001-June 2003 to general medicine services at the six hospitals. Patients were quasi-randomized to medical teams based on a day of the week-based call schedule or by the last digit of the patient's medical record number, depending on the site. If patients were unable to provide consent due to cognitive impairment, consent was sought through designated proxy. ${ }^{13}$ Patients were excluded from this part of the study if they were transferred to another service before discharge or died during hospitalization. Patients previously enrolled in the study were eligible for subsequent inclusion. Only patients enrolled in the study who were discharged alive from hospital and completed a follow-up phone call were included in the analyses. All patients, regardless of whether they were assigned to hospitalist or non-hospitalist care, were eligible for the study. Hospitalist physicians were defined by the individual institutions. In general, this corresponded to at least 2-3 months per year of ward clinical service with the majority performing 3 to 6 months, depending on the individual site. The non-hospitalist physicians were generally internal medicine subspecialists and outpatient-based general internists.

\section{Patient Follow-up and Outcome}

Patients or their proxies were telephoned 30 days after hospital discharge and asked about readmission to hospital or ED visits after discharge. Re-admission or ED visit could be to the same or a different hospital. Patients were also linked to the National Death Index (NDI) to assess for 30-day mortality. The primary composite outcome of interest was death, hospital readmission, or ED presentation within 30 days from hospital discharge.

\section{PCP Follow-up}

Patients identified their PCP during an inpatient interview, and then a random sample of these patients' primary care providers (PCPs) was surveyed via a faxed questionnaire 2 weeks after patient discharge. Depending on the site, the PCP for one in two (UNM), one in five (UC, UW), or one in ten (BWH, UCSF, UI) study patients discharged from the hospital was surveyed. This differential sampling was done because of limited resources at some centers and because of the prior knowledge that fewer patients at UNM had PCPs. The PCP survey was developed from reviewing relevant themes from the literature after an extensive literature search was unable to identify any suitable pre-existing instruments (Appendix). It was pilot tested on primary care providers at the University of Chicago prior to the study. The survey examined attitudes concerning the care and quality of communication provided by the inpatient physician, as well as whether or not the PCP had been aware of the patient's admission. The survey included 12 questions and asked about aspects of the communication, including the specific member of the team (e.g., nurse, medical student, attending physician), the timing of the communication (e.g., at admission, during the hospitalization, at discharge), and the type of method (e.g., phone, fax, or e-mail). The questions regarding the quality of communication were rated on a five-point scale (excellent, very good, good, fair, and poor). Two reminders were sent to non-responders.

\section{Statistical Analysis}

We used hierarchical, multi-variable, logistic regression techniques to model the association between communication with the patient's PCP and a 30-day composite outcome of ED visit, hospital readmission, or death adjusting for patient age, race, sex, Charlson comorbidity score, and study site. ${ }^{14}$ We also accounted for whether the patient's attending physician was defined by their institution as a hospitalist. Odds ratios were calculated compared to the reference group of patients with PCPs not aware of their hospitalization. Power calculations demonstrated that we had $79 \%$ power to detect a $9 \%$ difference for our primary question.

\section{RESULTS}

\section{Patient Description}

During the study period, 2,526 patients consented to have their PCP contacted, and 2,336 of these had valid PCP contact information. From these patients, 1,772 PCPs were contacted, and 1,213 responded (68\% response rate). We then excluded 423 patients who were lost to follow-up after discharge, leaving 
1,078 patients for analysis. The patient's inpatient attending physician was a hospitalist in $34 \%(371 / 1,078)$ of patients.

Patients and their hospitalizations are described in Table 1. Patients of PCPs who responded to the PCP survey had a mean age of 59 years, were mostly female, had a mean administrativedata-derived Charlson score of 1.1 (SD 1.5), and were mostly white. Patients of PCP respondents differed little from PCP nonrespondents except that they more commonly experienced the composite outcome and were less likely to be African-American.

\section{PCP Communication}

Overall, the PCPs for 834 patients (77\%) were aware that their patient was admitted to the hospital. There was no statistically significant difference in the PCP's knowledge of admission between hospitalist and non-hospitalist attendings (81\% vs. $78 \% ; \mathrm{P}=0.2$ ). Of those PCPs aware of the admission, there was direct communication with the general medicine service for 194 (23\%) patients, and 347 (42\%) PCPs reported seeing a discharge summary by 2 weeks after patient discharge.

\section{Patient Outcomes}

Within 30 days of discharge, 82 (7.6\%) patients died, 116 $(10.8 \%)$ patients were readmitted to hospital, and $69(6.4 \%)$ patients visited an ED. The composite outcome occurred in a total of 233 (22\%) patients: 49 (20\%) when the PCP was not aware of the hospitalization and 184 (22\%) when the PCP was aware of the hospitalization. (Table 2)

\section{Adjusted Analysis}

Multi-variable analysis found that PCP awareness of their patient's index admission to hospital was not associated with risk of the composite outcome (adjusted odds ratio 1.08, 95\%

Table 1. Comparison of Patient Demographics and Outcomes for Non-responder and Responder Primary Care Providers

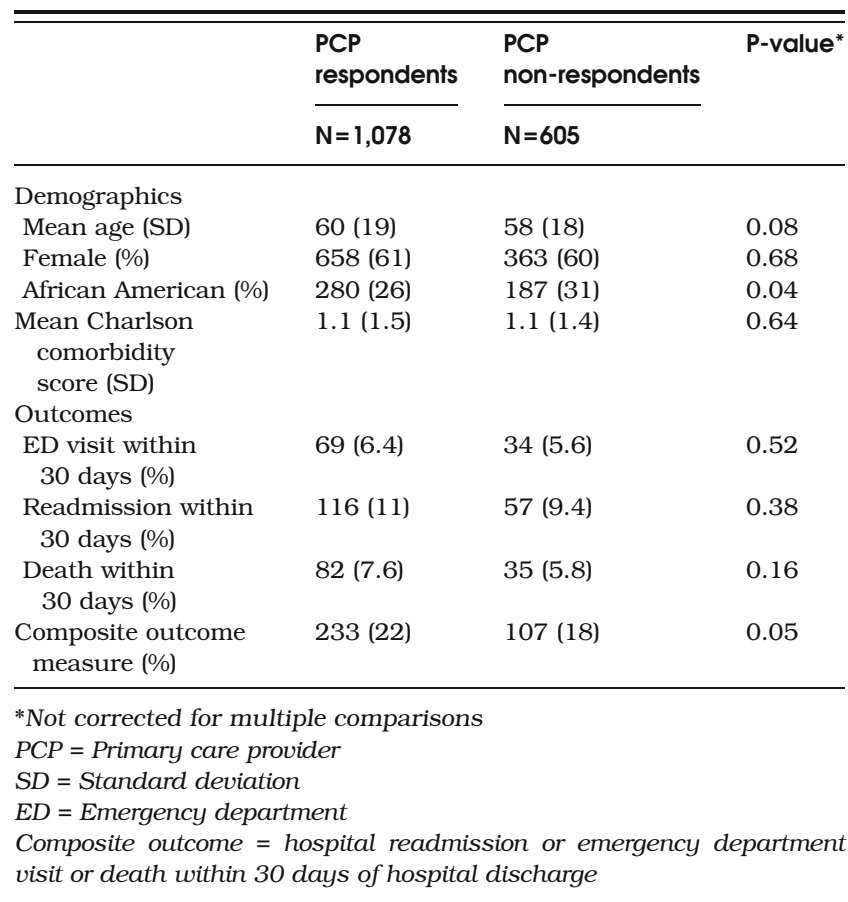

Table 2. Thirty-day Outcomes and PCP Communication

\begin{tabular}{|c|c|c|c|c|}
\hline \multirow[t]{2}{*}{ Survey question } & \multicolumn{3}{|c|}{ 30-Day outcomes } & \multirow[b]{2}{*}{$\begin{array}{l}\text { Composite } \\
\text { outcome (\%) }\end{array}$} \\
\hline & $\begin{array}{l}\text { Readmission } \\
(\%)\end{array}$ & $\begin{array}{l}\text { ED visit } \\
(\%)\end{array}$ & $\begin{array}{l}\text { Death } \\
(\%)\end{array}$ & \\
\hline $\begin{array}{l}\text { PCP not aware of } \\
\text { hospitalization } \\
(n=244)\end{array}$ & $21(8.6)$ & $19(7.7)$ & $18(7.3)$ & $49(20)$ \\
\hline $\begin{array}{l}\text { PCP aware of } \\
\text { hospitalization } \\
(n=834)\end{array}$ & $95(11.4)$ & $51(6.1)$ & $62(7.4)$ & $184(22)$ \\
\hline \multicolumn{5}{|c|}{$\begin{array}{l}\text { Among those patients whose PCPs were aware of hospital admission: } \\
\text { Communicated with inpatient team }\end{array}$} \\
\hline No $(\mathrm{n}=640)$ & $79(12.4)$ & $56(8.8)$ & $53(8.3)$ & $166(26)$ \\
\hline Yes $(n=194)$ & $22(11.2)$ & $11(5.5)$ & $14(7.4)$ & $41(21)$ \\
\hline \multicolumn{5}{|c|}{ Seen discharge summary } \\
\hline No $(n=487)$ & $59(12.1)$ & $30(6.1)$ & $32(6.6)$ & $112(23)$ \\
\hline Yes $(n=347)$ & $36(10.4)$ & $24(6.9)$ & $28(8.1)$ & $73(21)$ \\
\hline
\end{tabular}

$E D=$ Emergency department

Composite outcome $=$ hospital readmission or emergency department visit or death within 30 days of hospital discharge

$\mathrm{PCP}=$ Primary care provider

confidence interval 0.73 to 1.59 ). Similarly, there were no significant differences in adjusted 30-day composite outcomes if the PCP communicated directly with the hospital team (adjusted OR $0.87,95 \%$ CI 0.56 to 1.34 ) or if the PCP saw a discharge summary (adjusted OR $0.84,95 \%$ CI 0.57 to 1.22 ). Whether the in-hospital attending physician was a hospitalist had no effect on the composite outcome (adjusted OR 0.99, $95 \% 0.71$ to 1.4 ).

\section{DISCUSSION}

In a multicenter study of hospitalists and non-hospitalists in six US academic medical centers, few primary care providers (PCPs) had direct communication with the inpatient medical team during their patients' hospitalizations, more than half reported not receiving a discharge summary within 2 weeks, and almost one quarter did not have any knowledge that their patients had been admitted at all. However, these lapses in communication were not associated with adjusted 30-day risk for death, hospital readmission, or emergency department visits.

Much has been discussed about the importance of discharge communication for hospitalized patients, but little work has evaluated "hard" outcomes. ${ }^{9}$ Most previous studies of inter-physician communication have focused on process measures or surrogate markers, such as decreased laboratory test repetition, reduced emergency department waits, improved glycosylated hemoglobin levels, and higher cancer screening rates. ${ }^{15-18}$ However, not all work has demonstrated improved patient care. ${ }^{19,20}$ Perhaps the largest study that evaluated communication between hospital-based and ambulatory-care physicians with respect to patient outcomes assessed almost 900 patients and found that discharge summaries were available to treating physicians in less than $25 \%$ of follow-up visits. ${ }^{21}$ However, adjusted analyses of 90-day risk of hospital readmission related to discharge summary availability were not significant (odds ratio $0.74 ; 95 \%$ CI 0.05 to 1.10 ). This study involved only a single center and only measured hospital readmission at 90 days as compared to a composite outcome of 30-day death, hospital readmission, or ED visit that is now the standard for many studies of quality. 
Although our observed rate of PCP-hospital-based physician communication showed that almost one-quarter of PCPs were unaware that their patient was hospitalized, it is far lower than the average described in a recent systematic review. ${ }^{9}$ Previous studies have documented that formal direct communication between hospital and primary care physicians within 2 weeks occurs less than two-thirds of the time (range 29-80\%) depending on the study and communication type. ${ }^{9}$ Our findings may be due to the expectation that residents contact PCPs at many of the sites. Further, rates observed in earlier work could be due to publication bias underscoring poor performance and may have preceded the recent and widespread adoption of the hospitalist movement, which emphasizes timely communication. ${ }^{1,2,4,5,11}$

The lack of a significant relationship between these aspects of communication and patient outcomes after discharge may be explained by several factors. First, the study may have been underpowered to detect such a relationship. The effects of direct communication and receipt of a discharge summary were associated with non-significant reductions in the composite outcome. That knowledge of the admission did not show even a non-significant trend towards improved outcomes may have been due to chance or to the fact that simple knowledge of admission may not be sufficient to improve outcomes. Also, PCPs may have other means of discovering that their patient was admitted to hospital and may take appropriate steps for follow-up. ${ }^{11}$ Second, the presence of any communication is not the same as receipt of high-quality communication, and only the latter may be capable of improving outcomes. Third, there are many other factors that can lead to ED visits, hospital readmission, or death besides presence and quality of communication, thus limiting our ability to find an effect. Fourth, patients may have misreported ED visits and hospital readmissions, leading to measurement bias towards the null. Fifth, there may be confounding by indication: inpatient physicians may be more likely to communicate with PCPs regarding patients who are particularly at high risk for adverse outcomes after discharge. We did not assess for this aspect - particularly because many of our patients were younger and had fewer comorbidities. Finally, there may be other patient confounders of the outcome that could not be adjusted for in our analyses.

Interestingly, we observed little difference in PCP responses or adjusted patient outcomes between patients cared for by hospitalists compared to other attending physicians. There may be several explanations for this finding. First, hospitalist physicians may not have that large of an effect on the patient outcomes measured in this study even though there may be other benefits. Second, the major role that residents have in patient care in academic centers may mitigate some of the effect of the attending physician. Also, many of the hospitalists in our study were relatively inexperienced, and it may take a while to hone communication skills and develop relationships with community PCPs. ${ }^{12}$ Finally, there may be systemic barriers to communication (e.g., incorrect PCP contact information) that apply equally to all attending physicians. Further research in this area is warranted.

Our study has limitations that merit mention. First, our study was not designed to determine how improved communication and information transfer might avoid adverse outcomes. Second, no sites involved in the study employed standardized templates for discharge summaries during the study, even though it is a recommended practice. ${ }^{9,22}$ Third, the surveys measured PCP knowledge of patient hospitalization at 2 weeks. This should have allowed enough time for any informal communication or the delivery of formal discharge summaries. However, PCPs may still have received information after the survey was completed but prior to their patient's follow-up visit. Fourth, our study only involved academic centers and medical teams involving medical housestaff. Although many community hospitals may have closer relationships with PCPs, an increasing number of community hospitals also rely on hospitalist models of care, and the findings may therefore apply. ${ }^{1,2,4}$ Finally, our composite outcome of 30-day death, hospital or ED admission is not the only relevant metric. We did not present any information on other important patient outcomes like adverse drug events, missed follow-up, quality of life, and patient satisfaction. ${ }^{10,23,24}$

Our findings are strengthened by several aspects of this study. We studied diverse patient groups from disparate geographic areas throughout the US. Moreover, we accounted for possible differences between these six sites in our analyses. Further, we included data on the availability of the discharge summary as well as other communication strategies that may be preferred by PCPs, such as telephone or e-mail. ${ }^{11}$ Our use of a composite outcome allowed us to increase the statistical power of our study while still incorporating relevant quality of care measures. Finally, we had a high response rate of $70 \%$, limiting the potential impact of respondent bias.

Patients are being discharged from hospitals quicker and sicker than in previous years. ${ }^{10,25-27}$ Consequently, adequate follow-up and care continuity increases in importance. Though our results provide no direct link between physician communication and important patient outcomes, they demonstrate that communication between hospital physicians and PCPs can be substantially improved.

Acknowledgements: The work reported here was supported by the Agency for Healthcare Quality (RO1 HS 10597, A Multi-center Trial of Academic Hospitalists, PI: David Meltzer, MD, PhD). Dr. Bell is the recipient of a New Investigator Award from the Canadian Institutes of Health Research Institute of Aging. Dr. Kaboli is supported by a Research Career Development Award from the Health Services Research and Development Service, Department of Veterans Affairs (RCD 03-033-1). Dr. Schnipper is supported by a Mentored Clinical Scientist Award (HL072806) from the National Heart, Lung and Blood Institute, National Institutes of Health. Dr. Wetterneck was supported by a Mentored Clinical Research Scholars Award from the National Center for Research Resources, National Institutes of Health (RR01764-01), and is currently funded on a Mentored Clinical Scientist Development Award from AHRQ KO8 HS17014-02. Dr. Arora is supported by the Hartford Geriatrics Health Outcomes Research Scholars Award and a CTSA K12 Scholars Award (KL2RR02500O) from the National Center for Research Resources. The views expressed in this article are those of the authors and do not necessarily represent the views of the Department of Veterans Affairs, National Center for Research Resources, or the National Institutes of Health. The funding agencies had no role in the design and conduct of the study; collection, management, analysis, or interpretation of the data; or preparation, review, or approval of the manuscript. The corresponding author had full access to all of the data in the study and takes responsibility for the integrity of the data and the accuracy of the data analysis. 


\section{Conflict of Interest: None disclosed.}

Corresponding Author: Chaim M. Bell, MD, PhD, FRCP(C); St. Michael's Hospital, 30 Bond Street, Toronto, ON M5B 1W8, Canada (e-mail: bellc@smh.toronto.on.ca).

\section{REFERENCES}

1. Wachter RM, Goldman L. The emerging role of "hospitalists" in the American health care system. N Engl J Med. 1996;335(7):514-7.

2. Wachter RM, Goldman L. The hospitalist movement 5 years later JAMA. 2002;287(4):487-94.

3. Lindenauer PK, Rothberg MB, Pekow PS, Kenwood C, Benjamin EM, Auerbach AD. Outcomes of care by hospitalists, general internists, and family physicians. N Engl J Med. 2007;357(25):2589-600.

4. Wachter RM. An introduction to the hospitalist model. Ann Intern Med. 1999; 130(4 Pt 2):338-42.

5. Alpers A. Key legal principles for hospitalists. Am J Med. 2001;111 (9B):5S-9S.

6. Goldman L, Pantilat SZ, Whitcomb WF. Passing the clinical baton: 6 principles to guide the hospitalist. Am J Med. 2001;111(9B):36S-9S.

7. Sox HC. The hospitalist model: perspectives of the patient, the internist, and internal medicine. Ann Intern Med. 1999;130(4 Pt 2):368-72.

8. van Walraven C, Seth $\mathbf{R}$, Laupacis A. Dissemination of discharge summaries. Not reaching follow-up physicians. Can Fam Physician. 2002;48:737-42.

9. Kripalani S, LeFevre F, Phillips CO, Williams MV, Basaviah P, Baker DW. Deficits in communication and information transfer between hospital-based and primary care physicians: implications for patient safety and continuity of care. JAMA. 2007;297(8):831-41.

10. Forster AJ, Murff HJ, Peterson JF, Gandhi TK, Bates DW. The incidence and severity of adverse events affecting patients after discharge from the hospital. Ann Intern Med. 2003;138(3):161-7.

11. Pantilat SZ, Lindenauer PK, Katz PP, Wachter RM. Primary care physician attitudes regarding communication with hospitalists. Am J Med. 2001;111(9B):15S-20S.

12. Meltzer D, Manning WG, Morrison J, et al. Effects of physician experience on costs and outcomes on an academic general medicine service: results of a trial of hospitalists. Ann Intern Med. 2002;137(11):866-74.
13. Roccaforte WH, Burke WJ, Bayer BL, Wengel SP. Validation of a telephone version of the mini-mental state examination. J Am Geriatr Soc. 1992;40(7):697-702.

14. Charlson ME, Pompei P, Ales KL, Mackenzie CR. A new method of classifying prognostic comorbidity in longitudinal studies: development and validation. J Chronic Dis. 1987;40(5):373-83.

15. Wagner E, Duggan MA. Effectiveness of follow up-letters to health care providers in triggering follow-up for women with abnormal results on Papanicolaou testing. CMAJ. 2001;164(2):207-8.

16. Robertson CL, Kopans DB. Communication problems after mammographic screening. Radiology. 1989;172(2):443-4.

17. Branger PJ, van 't HA, van der Wouden JC, Duisterhout JS, van Bemmel JH. Shared care for diabetes: supporting communication between primary and secondary care. Medinfo. 1998;9(Pt 1):412-6.

18. Montalto $\mathbf{M}$, Harris $\mathbf{P}$, Rosengarten $\mathbf{P}$. Impact of general practitioners' referral letters to an emergency department. Aust Fam Physician. 1994;23(7):1320-25. 1328.

19. Crone P. Are preadmission general practitioner telephone calls of value? A study in communication. N Z Med J. 1987;100(833):632-4.

20. Ferguson JA, Goldacre MJ, Henderson J, Gillmer MD. Audit of workload in gynaecology: analysis of time trends from linked statistics. Br J Obstet Gynaecol. 1991;98(8):772-7.

21. van Walraven C, Seth R, Austin PC, Laupacis A. Effect of discharge summary availability during post-discharge visits on hospital readmission. J Gen Intern Med. 2002;17(3):186-92.

22. Joint Commission on Accreditation of Healthcare Organizations. Joint Commission national patient safety goals. 2007. Ref Type: Report.

23. Roy CL, Poon EG, Karson AS, et al. Patient safety concerns arising from test results that return after hospital discharge. Ann Intern Med. 2005; 143(2):121-8.

24. Forster AJ, Murff HJ, Peterson JF, Gandhi TK, Bates DW. Adverse drug events occurring following hospital discharge. J Gen Intern Med. 2005;20(4):317-23

25. Kosecoff J, Kahn KL, Rogers WH, et al. Prospective payment system and impairment at discharge. The 'quicker-and-sicker' story revisited. JAMA. 1990;264(15):1980-3.

26. Halm EA, Magaziner J, Hannan EL, et al. Frequency and impact of active clinical issues and new impairments on hospital discharge in patients with hip fracture. Arch Intern Med. 2003;163(1):108-13.

27. Baker DW, Einstadter D, Husak SS, Cebul RD. Trends in postdischarge mortality and readmissions: has length of stay declined too far. Arch Intern Med. 2004;164(5):538-44. 


\section{APPENDIX. PRIMARY CARE PROVIDER SURVEY}

To the primary health-care provider of the above patient:

\section{To the primary healthcare provider of the above patient:}

1. Were you aware that this patient had been hospitalized? Yes__ No

[IF YOU ANSWERED NO, PLEASE SKIP TO QUESTION \#9]

2. Did you communicate with the medical team caring for this patient? If so, when?

No. I was aware this patient was hospitalized, but there was no communication with the medical team.

[IF YOU ANSWERED NO, PLEASE SKIP TO QUESTION \#3]

Yes, there was communication with the medical team. (Please check all that apply.)

At admission:

_initiated by me

initiated by medical team

During hospitalization:

_ initiated by me initiated by medical team

At discharge:

__ initiated by me initiated by medical team

After discharge:

__ initiated by me initiated by medical team

2a. What members of the medical team did you communicate with? (Please check all that apply.)

Nurse: Medical student: Resident/Fellow: PA/NP: Attending: Donít know:

2b. In what ways have you communicated with the medical team who cared for this patient? (Please check all that apply.)

by Telephone: by E-mail: in Person: by Fax: by Mail: Not Applicable:

2c. How would your rate the communication with the medical team who cared for this patient?
Excellent
Very Good:
Good:
Fair:
Poor:

3. Did you communicate with this patient or his/her family about the hospitalization? If so, when?

_ No, I was aware this patient was hospitalized, but there was no communication with the patient or his/her family about the hospitalization.

_ Yes, there was communication with the patient or his/her family. (Please check all that apply.)

At admission:

During hospitalization:

At discharge:

After discharge:

4. In what ways have you communicated with the patient or his/her family about the hospitalization? (Please check all that apply.)

by Telephone: by E-mail: in Person: by Fax: by Mail: Not Applicable:

5. Have you seen a discharge summary on this patient for this hospitalization?

Yes:

No, but had access to one online:

No:

Don't Know:

[IF YES, CONTINUE; OTHERWISE PLEASE SKIP TO QUESTION \#6]

5a. How would you rate the completeness and organization of the discharge summary?
Excellent:
Very Good:
Good:
Fair:
Poor:

5b. How would you rate the timeliness of the discharge summary?
Excellent:
Very Good:
Good:
Fair:
Poor: 